\title{
A Full-Scale Experimental Validation of Electromagnetic Time Reversal Applied to Locating Disturbances in Overhead Power Distribution Lines
}

\author{
Zhaoyang Wang, Student Member, IEEE, Shaoyin He, Qi Li, Buying Liu, Reza Razzaghi, Member, \\ IEEE, Mario Paolone, Senior Member, IEEE, Yanzhao Xie, Member, IEEE, Marcos Rubinstein, \\ Fellow, IEEE, and Farhad Rachidi, Fellow, IEEE
}

\begin{abstract}
Electromagnetic time reversal (EMTR) has emerged as a promising technique to locate disturbances in power grids thanks to its location accuracy and robustness against parameters uncertainties. Furthermore, in a reflective medium, like the one of a power network, it has been shown that the method requires no more than one single observation point. In this paper, we present an experimental validation of EMTR to locate disturbances in real power networks. The validation is performed on a full-scale unenergized 677-m long, double-circuit $10-\mathrm{kV}$ overhead distribution line. The disturbance is emulated by a voltage pulse injected between one of the line conductors and the ground using a high voltage pulse generator. The frequency spectrum of the injected voltage pulse is specified such that the originated electromagnetic transients are compatible with those of power line faults, lightning and conducted intentional electromagnetic interferences. The transient currents generated by the emulated disturbance are measured at one end of the line, considering two different line configurations. According to the EMTR technique, the measured signals are time reversed and back injected into the system that, in our case, is a simulated model of the considered distribution line. More specifically, it is represented by a constantparameter line model implemented within the EMTP-RV simulation environment. For both cases, the disturbance is accurately located, and the phase of the circuit at which the pulse was injected is also identified.
\end{abstract}

Index Terms - electromagnetic time reversal, electromagnetic transients, distribution lines, disturbance location, power system protection.

\section{INTRODUCTION}

$\mathrm{P}$ OWER networks (both transmission and distribution) are subjected to a range of unexpected momentary voltage

This work has been supported by the Swiss Competence Center for Energy Research FURIES (Future Swiss Electrical Infrastructure) and the National Natural Science Foundation of China under Grant 51677148.

Z. Wang, M. Paolone, and F. Rachidi are with the Electromagnetic Compatibility Laboratory and the Distributed Electrical Systems Laboratory, Swiss Federal Institute of Technology Lausanne, Lausanne 1015, Switzerland (e-mail: zhaoyang.wang@epfl.ch; mario.paolone@epfl.ch; farhad.rachidi@epfl.ch).

S. He, Q. Li, B. Liu and Y. Xie are with the State Key Laboratory of Electrical Insulation and Power Equipment, School of Electrical Engineering, Xi'an variations from both natural and man-made disturbances [1]-[3]. The former category of disturbances is mainly originated by either direct or indirect lightning strikes (e.g., [4], [5]) or weather-induced shunt faults (e.g., ice, falling trees), unintentional contact to power lines by flying objects or animals, etc. [6], [7]. Man-made disturbances refer to switching operations either of network components, or end-user equipment (e.g., [1]-[3]), and, within the contemporary scene, acts of sabotage, such as conducted intentional electromagnetic interference (IEMI) [8], [9]. In general, the above-mentioned disturbances cause adverse effects on the quality of power supply with manifestations ranging from voltage sags and swells, insulation deterioration, sensitive load damage and power supply interruptions and even blackouts.

In this respect, the problem of distribution networks protection and, more generally, quality of power supply has historically received great attention with the aim of developing methods to protect network terminal components (e.g., distribution transformers) as well as power lines and cables [10]-[12].

In view of the above, identifying the location of the source of a generic disturbance is certainly a desired feature in any protection scheme in power systems. More specifically, among the previously-described disturbances, locating lightning strikes, faults, and electromagnetic pulse (EMP) injection is especially needed for coordinated protective maneuvers such as relay operation and fault clearance.

For the specific case of surges due to direct or indirect lightning, a major concern is related to overvoltages causing insulation deterioration and failures of power network components, especially at the location of the surge appearance (i.e., direct lightning strikes) as well as for nearby towers where

Jiaotong University, Xi'an 710049, China (e-mail: heshaoyin8421@stu.xjtu. edu.cn; yzxie@mail.xjtu.edu.cn).

R. Razzaghi is with the Department of Electrical and Computer Systems Engineering, Monash University, Melbourne 3800, Australia (e-mail: reza. razzaghi@monash.edu).

M. Rubinstein is with the University of Applied Sciences of Western Switzerland, Yvderon-les-bains 1400, Switzerland (e-mail: marcos. Rubinstein (a)heig-vd.ch). 
(back-) flashovers are likely to arise [4], [13]. Identifying the locations of these flashovers greatly contributes to reducing time and labor consumption associated with insulation inspection and maintenance.

On the other hand, shunt faults are triggered by undesired electrical connections (mainly referring to phase(s)-to-ground cases) and require, in general, manual clearance. Therefore, indicating fault locations in power networks is needed for an adequate reconfiguration of the networks and expediting the power system restoration.

Finally, the use of high power electromagnetic sources to produce interferences in critical power system infrastructures (e.g., terminal power transformer and its secondary circuits in distribution networks) has become a serious concern. There is an increasing interest in detecting and locating IEMI attacks [14], [15].

Recently, the theory of electromagnetic time reversal (EMTR) has been applied to locate different types of disturbances in power systems and electromagnetic compatibility (EMC) applications [16]. The considered applications include locating lightning flashes (e.g., [17]), lightning-originated flashovers (e.g., [18]), short-circuit faults (e.g., [19]-[23]), and soft faults (e.g., [24], [25]). As known, the EMTR methods take advantage of the time reversibility of wave equations and the spatial correlation property of the time reversal theory to refocus the time-reversed back-propagated electromagnetic waves into the original disturbance location. More specifically, when the observed disturbance-originated electromagnetic transients are time reversed and back injected into the original system, they refocus back to the location of the disturbance.

In this paper, we present the results of a field experiment demonstrating the ability of an EMTR-based location method to identify the location of an injected disturbance along an overhead power distribution line. This experiment was performed on a 677-m long, $10-\mathrm{kV}$ distribution line in Yuncheng city, Shanxi Province, China.

The paper is structured as follows. Section II summarizes the basic principles of EMTR and its application for locating disturbances in power system. In Section III, we present the experimental setup and validation approach. Experimental results associated with two different configurations are presented and discussed in Section IV. Finally, Section V concludes this paper with final remarks.

\section{EMTR-BASEd Disturbance Location Method}

\section{A. EMTR and time-reversal invariance of transmission-line equations}

A system is time-reversal invariant with respect to a physical quantity if, given a solution $f(t)$ to its underlying equations, the time-reversed function $g(t)$, given by (1) hereunder, is a solution too [26].

$$
g(t)=f(-t)
$$

In order to ensure the causality requirements, the timereversed function $f(-t)$ is shifted in time as

$$
g(t)=f(-t+T)
$$

where, $T$ is the time window during which $f(t)$ is observed.

The time-reversal invariance is readily described and universally embodied in most laws of nature, either in a strict or in a soft sense [26]. Time reversal as a focusing process, first has been theoretically and experimentally studied in the field of acoustics (e.g., [27]-[29]). Later, its application was extended to electromagnetics using the acronym electromagnetic time reversal (EMTR).

Based on the transmission line theory, the propagation of voltage and current waves along the lines is governed by the Telegrapher's equations. With particular reference to the Telegrapher's equation describing the current wave propagation along multi-conductor lossless transmission lines, the current wave equation reads

$$
\frac{\partial^{2}}{\partial x^{2}} \boldsymbol{I}(x, t)-\boldsymbol{C} \boldsymbol{L}^{\prime} \frac{\partial^{2}}{\partial t^{2}} \boldsymbol{I}(x, t)=\mathbf{0}
$$

where $\boldsymbol{I}(x, t)$ is the vector of currents along the lines, and $\boldsymbol{C}^{\prime}$ and $\boldsymbol{L}^{\prime}$ are the per-unit-length matrices of capacitances and inductances, respectively.

Because of the second-order time derivative, if $\boldsymbol{I}(x, t)$ is a solution of (3), $\boldsymbol{I}(x,-t+T)$ is also a solution, namely

$$
\frac{\partial^{2}}{\partial x^{2}} \boldsymbol{I}(x,-t+T)-\boldsymbol{C}^{\prime} \boldsymbol{L}^{\prime} \frac{\partial^{2}}{\partial t^{2}} \boldsymbol{I}(x,-t+T)=\mathbf{0}
$$

The same applies to the voltage equation. This implies that the wave equation for a lossless transmission-line system is time reversal invariant. Furthermore, it has been recently shown that the method's performance in terms of location accuracy is not affected in the case of lossy lines for which the wave equations are not rigorously time-reversal invariant [30].

\section{B. Applying EMTR to locate disturbances in power networks}

Benefiting from the time reversal invariance of the transmission-line equations and the inherent spatial focusing property of the time-reversal invariant system, EMTR has emerged as an extremely promising technique of detecting and locating various disturbances in power systems.

Theoretically, direct lightning strikes, faults and conducted IEMI disturbances can be represented by an equivalent voltage or current source with specified characteristics (e.g., frequency spectrum) according to each type of disturbance [8], [19], [31].

Locating the source of the disturbance using an EMTR-based method is carried out in two successive steps named direct-time and reversed-time.

Since the focus of this paper is to provide an experimental validation, in the direct time, a voltage or current source is intentionally connected to a specific location along the line (e.g., $x=x_{f}$ ), emulating the occurrence of the disturbance at that location. The generated electromagnetic transient (EMT) signals are measured at a single observation point located at the 
line terminal, on one of the phases or on all of the phases depending on the experiment.

In the reversed time, the measured transient signals are time reversed and synchronously back-injected from the respective observation point into the network model using numerical simulations. The simulations are performed for different guessed positions of the original disturbance along the line. At the point corresponding to the real location, the two media in the direct and reverse phases match and, therefore, the backinjected signals refocus at the original disturbance location.

Different metrics can be used to quantify the wave refocusing property [19], [22]-[25]. In this paper, we will use the metric proposed in [19] that analyzes, as a function of the guessed location, the energy of the signal of the shunt current flowing in a generic location of the disturbance. It has been demonstrated that the transverse branch current signal observed at the true location presents a maximum energy compared to any other location along the line [18]-[21]. This method has been successfully used to locate faults (e.g., [19]-[21]) and lightning flashovers (e.g., [18]]) along power networks.

In the next section, we will present a full-scale experimental validation of the EMTR-based method to locate disturbances in power lines, by making use of a real overhead power distribution line.

\section{EXPERIMENTAL SETUP AND APPROACH}

In November 2016, a field experiment was performed on an unenergized $10-\mathrm{kV}$ three-phase distribution line in Shanxi Province, China, with the objective of validating the capability of the EMTR-based technique to locate a disturbance incident in a real power system environment. In this section, we will introduce the setup and the approach proposed for this experiment.

\section{A. Experimental setup}

The transmission-line system considered in the field experiment is a $10-\mathrm{kV}$ double-circuit overhead power distribution line. The cross-sectional geometry and main electrical parameters of the overhead line are respectively described in Fig. 1 and Table I.

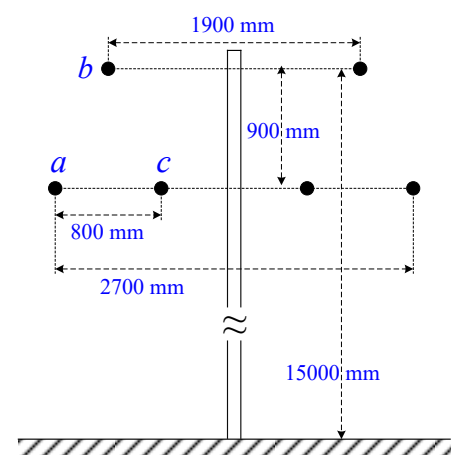

Fig. 1. Cross-sectional geometry of the $10-\mathrm{kV}$ double-circuit overhead power distribution line.
TABLE I

MAIN ELECTRICAL PARAMETERS OF THE DistRIBUTION LINE

\begin{tabular}{cc}
\hline \hline Parameter & Value \\
\hline $\begin{array}{c}\text { Diameter of the line conductor } \\
\text { Conductivity of the line conductor }\end{array}$ & $18.4 \mathrm{~mm}$ \\
Soil conductivity & $3.5 \times 10^{7} \mathrm{~S} / \mathrm{m}$ \\
(aluminum conductor steel reinforced (ACSR)) & $0.1 \mathrm{~S} / \mathrm{m}$ \\
\hline \hline
\end{tabular}

It should be noted that, in the direct-time phase, the system is unenergized, and the disturbance emulation and measurement were performed by making use of one of the two three-phase single-circuits (see the three-phase conductors indicated by $a, b$ and $c$ in Fig. 1). Another circuit of the line (i.e., the one on the right-hand side in Fig. 1) was left open at both extremities during the experiment. It is worth mentioning that both circuits were included in the reversed-time simulation by taking the existing electromagnetic coupling into consideration.

The experimental setup is illustrated in Fig. 2. As shown, the considered overhead-line section is characterized by a total line length of $677 \mathrm{~m}$, including 11 overhead-line towers (numbered from 22 to 32). To emulate a disturbance occurrence, a voltage pulse generator was connected between the phase $c$ of the overhead line and the ground at tower No. 23 (see Fig. 3) situated $68 \mathrm{~m}$ away from the origin of the line. The injected pulse signal $V_{p}(t)$ is characterized by a risetime $t_{r}$ of $12 \mathrm{~ns}$, and a full width at half maximum (FWHM) of about $1 \mu \mathrm{s}$.

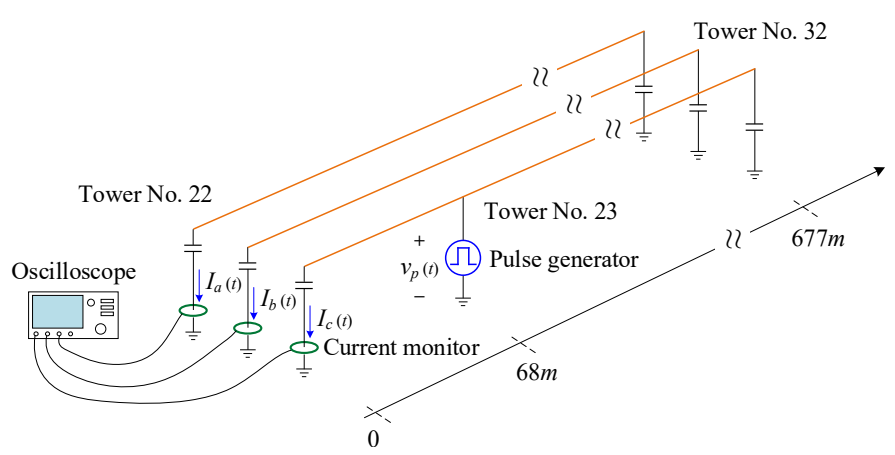

Fig. 2. Schematic representation of the experimental setup. Another three-phase circuit (not shown in this diagram) was in open-circuit at both ends.

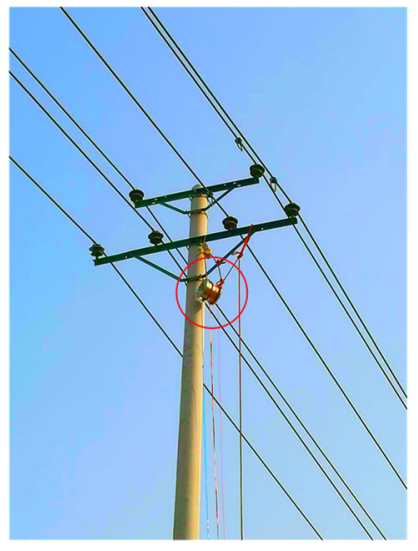

Fig. 3. Picture of Tower No. 23 at which the pulse generator (marked with the red circle) was connected to one of the phase conductors of the overhead line. 
In reality, the power network is typically terminated with power transformers. The high-frequency input impedance of power transformer can be represented, to a first approximation, by its winding-to-ground capacitance $C_{t}$ [32], [33]. Additionally, the power transformers are connected to the substation bus through the bushing, whose electrical characteristics are also dominated by a line-to-ground capacitance $C_{b}$ during the power transient phenomena of interest. Therefore, the port-characteristics of the transformer (seen from the incoming-line side) can be equivalently represented by a parallel circuit of $C_{t}$ and $C_{b}$.

According to the actual measurements on $10-\mathrm{kV}$-level transformers operating at the local power network, the capacitances $C_{t}$ and $C_{b}$ were found to be in the range $800 \mathrm{pF}$ to $900 \mathrm{pF}$, and $450 \mathrm{pF}$ to $550 \mathrm{pF}$, respectively. As a result, we used $1500 \mathrm{pF}$ thin-film capacitors to represent the $10-\mathrm{kV}$-level terminal power transformers in the field experiment (see Fig. 2).

One of the promising properties of time-reversal theory in a reflective medium is that refocusing is possible using single end/side observation [19], [27]-[29]. As a result, in the field experiment, the disturbance-originated transients were recorded at the left line end (tower No. 22) by means of broadband current monitors (see Fig. 2). The measurement devices and their main characteristics are summarized in Table II.

TABLE II

MEASUREMENT DEVICES AND THEIR CHARACTERISTICS

\begin{tabular}{cccc}
\hline \hline Device & Model & Parameter & Value \\
\hline \multirow{2}{*}{ Current monitor } & Pearson $^{\mathrm{TM}}$ & Sensitivity & $0.5 \mathrm{~V} / \mathrm{A}$ \\
& $8585 \mathrm{C}$ & Bandwidth & $200 \mathrm{MHz}$ \\
\hline \multirow{3}{*}{ Oscilloscope } & \multirow{2}{*}{ Tektronix $^{\mathrm{TM}}$} & Sampling rate & $2.5 \mathrm{GS} / \mathrm{s}$ \\
& DPO 3054 & Vertical resolution & $11 \mathrm{bits}$ \\
& & Bandwidth & $500 \mathrm{MHz}$ \\
\hline \hline
\end{tabular}

\section{B. Disturbance emulation}

In this work, the disturbance is emulated by a voltage pulse injected between one of the line conductors and the ground using a pulse generator. The waveform of the injected voltage has been selected so that its frequency spectrum covers those of the electromagnetic transients caused by power line faults, lightning and conducted IEMI. The frequency spectrum of transients generated by faults in power networks extends to some hundreds of $\mathrm{kHz}$, while lightning-induced overvoltages have significant frequency components up to a few $\mathrm{MHz}$ [2]. Conducted IEMI pulses can be characterized by diverse waveshapes. However, due to the high-frequency propagation losses along power lines, the frequency spectrum of these sources generally does not extend beyond $10 \mathrm{MHz}$ or so [8].

As a result, a compact capacitor-discharging pulse generator was adopted in the study (see Fig. 4a). The pulse generator can be equivalently represented by a simple $R L C$ circuit (see Fig. 4b), in which the capacitance $C$ storing the energy to produce the surge has a value of $6.97 \mathrm{nF}$. The coaxial structure of the generator restricts the discharging-circuit inductance $L$ to some hundreds of $\mathrm{nH}$. The resistance $R$ in the circuit represents the equivalent impedance seen by the generator when connected to the overhead line. More specifically, in the considered experimental configuration, $R$ corresponds to $Z_{c} / 2$, $Z_{c}$ being the characteristic impedance of phase $c$ of the circuit, which is about $400 \Omega$. Note that this is an approximated computation since it is ignoring the presence of the other mutual coupling between the conductors. In addition, the discharging voltage of the generator is controlled by a spark gap switch (see $S$ in Fig. 4b). Compressed air is used as dielectric in the switch.

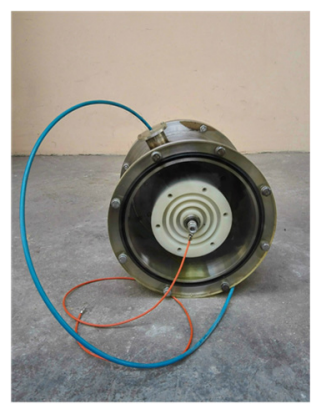

(a) Picture

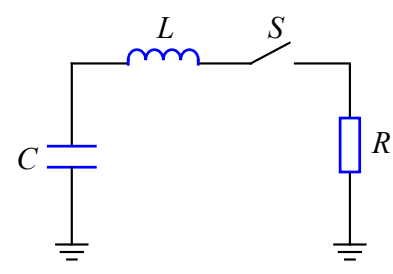

(b) Equivalent circuit
Fig. 4. Picture and equivalent circuit of the adopted coaxial-structure pulse generator

In the laboratory environment, we used a $200 \Omega$ metal oxide film resistor to simulate the discharging condition of the field experiment. As shown in Fig. 5a, the measured waveform of the voltage pulse presents, as mentioned earlier, a risetime of about $12 \mathrm{~ns}$ and an FWHM of about $1 \mu \mathrm{s}$. The spectrum of the pulse extends to significant frequencies, up to a few tens of $\mathrm{MHz}$ (see Fig. 5b).

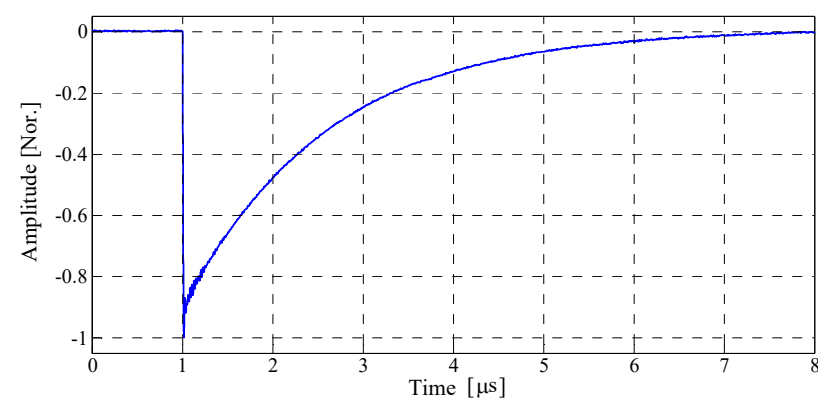

(a)

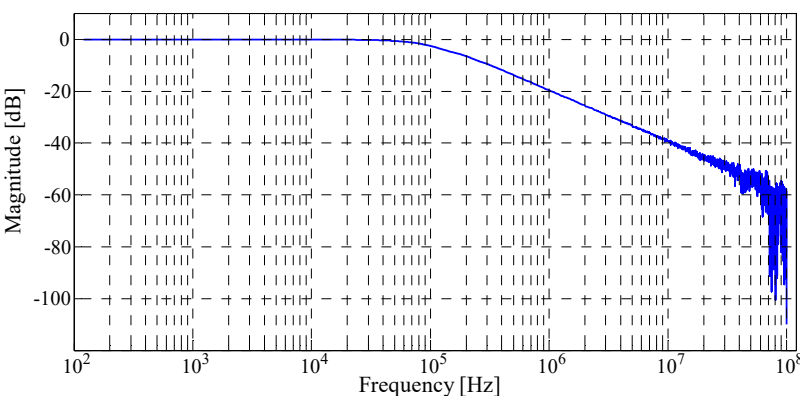

(b)

Fig. 5. Normalized waveform (a) and magnitude spectrum (b) of the voltage pulse characterized by a risetime and an FWHM of 12ns and $960 \mathrm{~ns}$ respectively. The spectral components appearing in the frequency exceeding $100 \mathrm{MHz}$ are not shown in this figure, as they are considered to be mainly due to quantization noise (sampling rate: $2.5 \mathrm{GS} / \mathrm{s}$ ). 


\section{EXPERIMENTAL RESULTS AND DISCUSSION}

Recalling the setup described in Fig. 2, we emulated a power system disturbance occurrence by injecting a voltage pulse signal $V_{p}(t)\left(t_{r}=12 \mathrm{~ns}, \mathrm{FWHM}=1 \mu \mathrm{s}\right)$ into the line conductor of phase $c$. The injection point was situated at the overhead-line tower No. 23, which is $68 \mathrm{~m}$ away from the observation point (OP), namely tower No. 22. The peak value of the injected pulse $V_{p}(t)$ was about $10 \mathrm{kV}$.

In what follows, we will present the experimental validation of the EMTR technique, considering two different line configurations.

\section{A. One-phase configuration}

\section{1) Direct-time measurements}

In the first experiment, only the injected phase $c$ was terminated at each end on a $1500 \mathrm{pF}$ thin-film capacitor, whereas the other two phase conductors ( $a$ and $b$ ) were left open, as shown Fig. 6.

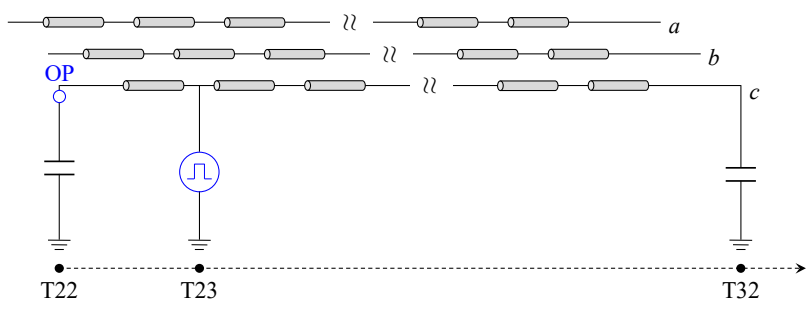

Fig. 6. Direct-time experimental setup of the one-phase configuration. The current transients generated by the source were measured only in the phase at which the disturbance pulse was injected. The second three-phase circuit (not shown in the diagram) was left open at both of its extremities.

The transient current signal generated by the source and measured at the observation point is shown in Fig. 7.

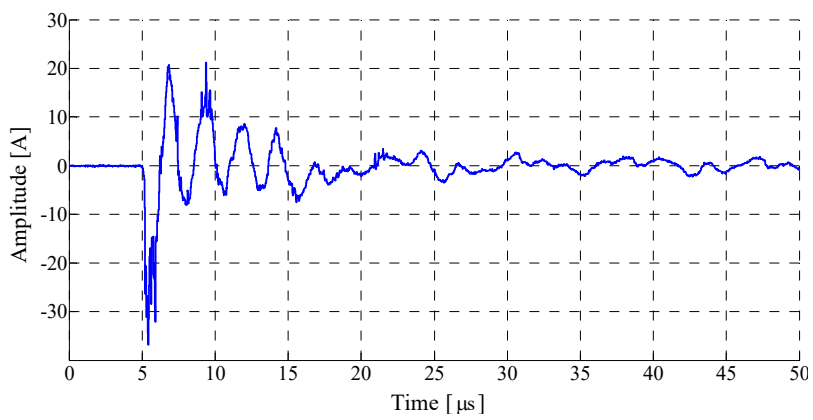

Fig. 7. Transient current waveform in phase $c$ measured at the observation point (sampling rate: $2.5 \mathrm{GS} / \mathrm{s}$ ).

\section{2) Reversed-time simulations}

In the reversed-time stage, the measured transients are time reversed and back injected numerically into a simulation model of the studied system.

According to its geometrical and electrical parameters, the line topology considered in the field experiments was modelled in the EMTP-RV environment by making use of the constantparameter (CP) line module [34], [35]. Note that, for achieving an accurate representation of the actual situation, both circuits of the 10-kV line (see Fig. 1) were considered in the simulation model. The generated per-unit-length parameters of the double circuits are given by (5)-(8), shown in Appendix.

As regards the line boundary conditions, the terminal element composed of the thin-film capacitor and its leading wires was modelled using an $R L C$ equivalent circuit (see Fig. 8), which was obtained through actual measurements by means of an impedance analyzer (i.e., Model 6500B of Wayne $\mathrm{Kerr}^{\mathrm{TM}}$ ) in a frequency range from $\mathrm{DC}$ to $20 \mathrm{MHz}$.

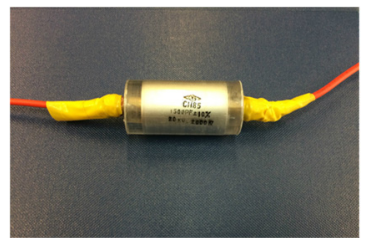

(a) Picture

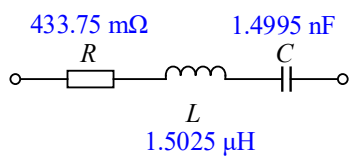

(b) $R L C$ circuit
Fig. 8. Picture and $R L C$ equivalent circuit of the terminal element composed of a thin-film capacitor with a nominal value of $1500 \mathrm{pF}$ and its leading wires.

In the simulations, after back-injecting the time-reversed transient current from the initial observation point (i.e., tower No. 22 in the EMTP-RV model), the transverse branch current was evaluated at different a priori guessed locations. In the presented line topology, we predefined the location of each tower as a guessed location, resulting in an average distance between two consecutive guessed locations is of $67.7 \mathrm{~m}$.

In Fig. 9, the normalized current energy is presented as a function of the guessed location. The normalization is conducted with reference to the maximum of the energy. In agreement with the EMTR-based method, the location of phase $c$ of tower No. 23 is evidently characterized by the highest energy concentration.

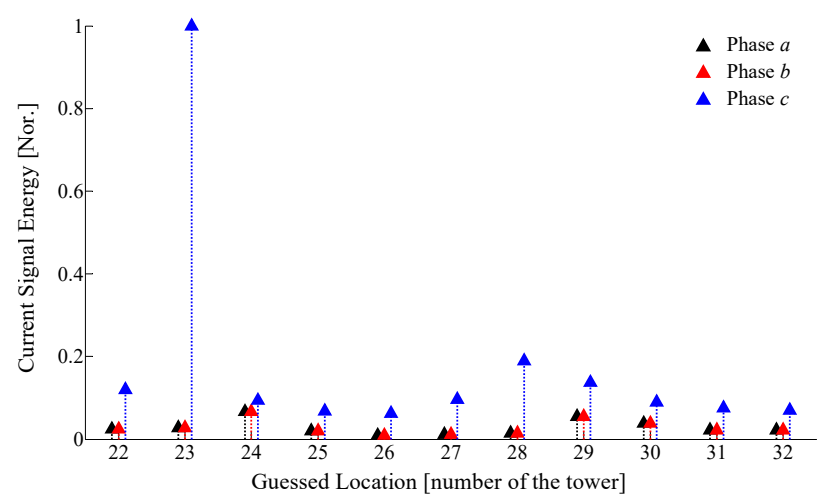

Fig. 9. Normalized energy of the branch current in the one-phase configuration.

\section{B. Three-phase configuration}

\section{1) Direct-time measurements}

In the second experiment, all three phases of the circuit were terminated at both ends on $1500 \mathrm{pF}$ thin-film capacitors. The transient currents in each phase conductor were simultaneously and synchronously recorded at the observation point (see OP1, $\mathrm{OP} 2$, and OP3 in Fig. 10). The pulse signal $V_{p}(t)$ was again injected into the phase $c$. The measured waveforms of the transient currents in the three phases are shown in Fig. 11. 


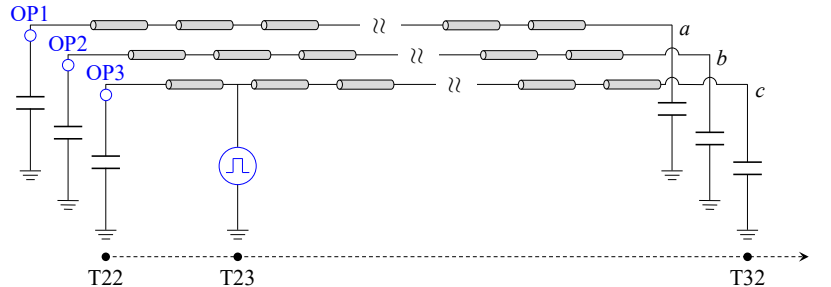

Fig. 10. Direct-time experimental setup of the three-phase configuration. The current transients generated by the source were measured in the three phases at the left line end. The second three-phase circuit (not shown in the diagram) was left open at both of its extremities.

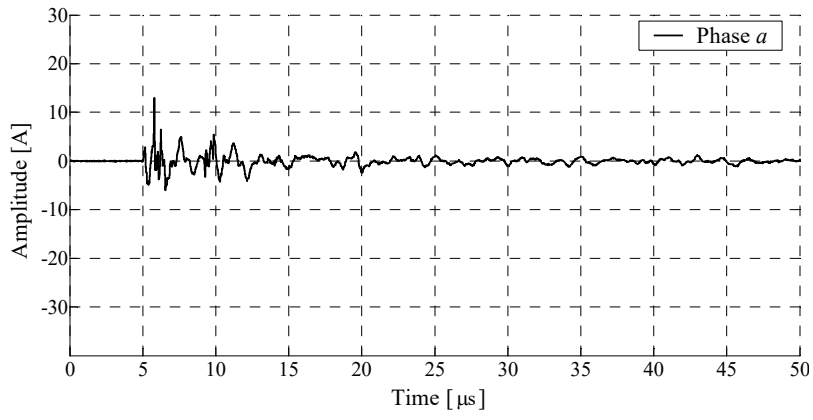

(a)

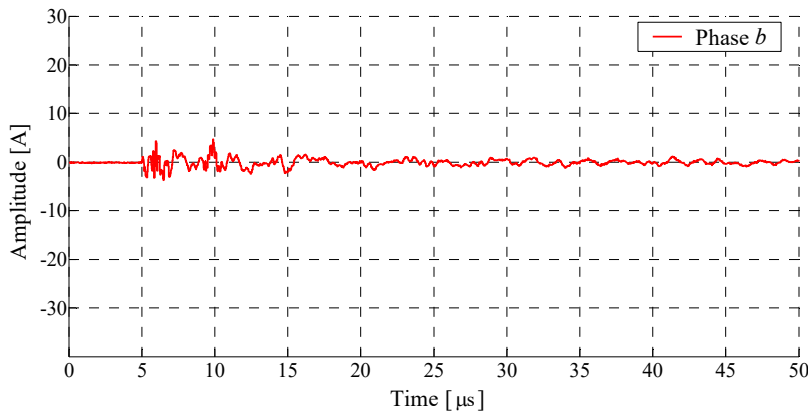

(b)

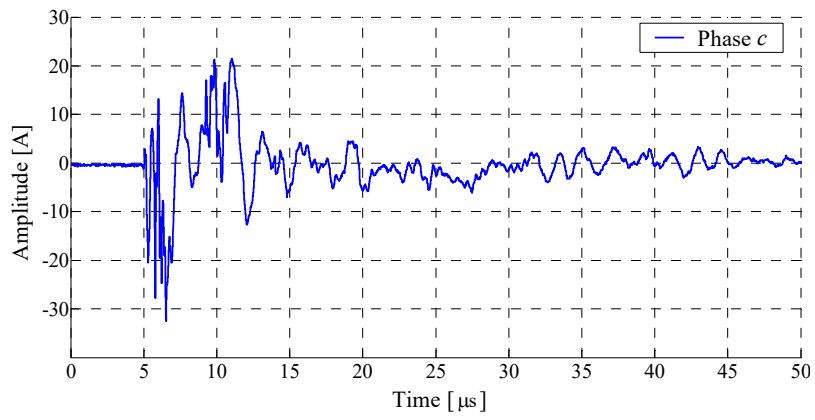

(c)

Fig. 11. Synchronously-measured transient current waveforms in the three phases (sampling rate: $2.5 \mathrm{GS} / \mathrm{s}$ ), (a) phase a, (b) phase $\mathrm{b}$ and (c) phase $\mathrm{c}$.

\section{2) Reversed-time simulations}

In the three-phase scenario, the back-propagation simulation is conducted by simultaneously injecting the time-reversed transient currents in each phase from their respective observation point.

Fig. 12 shows the normalized branch current energy along the three-phase line (here the normalization has also been implemented by considering the maximum current energy). As can be observed, at each tower location, phase $c$ presents a dominant current energy accumulation, compared to the other two phases. More importantly, a maximum is clearly reached at tower No. 23 of phase $c$, at which the pulse $V_{p}(t)$ was originally injected. Therefore, the method is capable of identifying the excited phase and the location of the disturbance along that phase.

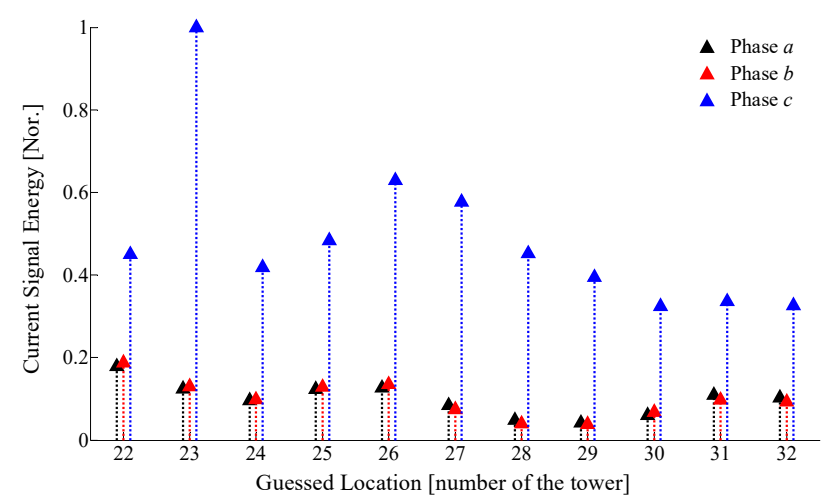

Fig. 12. Normalized energy of the branch current in the three-phase configuration.

\section{CONCLUSION}

We presented a full-scale experiment with the purpose of validating the EMTR-based method applied to locate power system disturbances. The pilot power network refers to a $677-$ $\mathrm{m}$ long $10-\mathrm{kV}$ double-circuit distribution line.

The disturbance was emulated by a voltage pulse injected between one of the line conductors and the ground using a pulse generator. The waveform of the injected voltage was selected so that its frequency spectrum covers those of power line faults, lightning and conducted IEMI subsequent transients.

The transient currents generated by the emulated disturbance were measured at one end of the line, considering two different line configurations. According to the EMTR technique, the measured signals were time reversed and back injected into an EMTP-RV constant-line model of the studied distribution line. For both cases, the disturbance was accurately located, and the conductor phase at which the pulse was originally injected was also identified.

To the best of the Authors' knowledge, this is the first experimental validation of the EMTR-based disturbance location technique in a real full-scale power line environment.

\section{APPENDIX}

The per-unit-length parameter matrices of resistance, $\boldsymbol{R}$, inductance, $\boldsymbol{L}$, conductance, $\boldsymbol{G}$, and capacitance, $\boldsymbol{C}$ of the considered double-circuit distribution line are shown below.

$\boldsymbol{R}=\left[\begin{array}{llllll}2.665 & 2.567 & 2.551 & 2.567 & 2.566 & 2.551 \\ 2.567 & 2.665 & 2.551 & 2.566 & 2.566 & 2.551 \\ 2.551 & 2.551 & 2.634 & 2.551 & 2.551 & 2.536 \\ 2.567 & 2.566 & 2.551 & 2.665 & 2.567 & 2.551 \\ 2.566 & 2.566 & 2.551 & 2.567 & 2.665 & 2.551 \\ 2.551 & 2.551 & 2.536 & 2.551 & 2.551 & 2.634\end{array}\right] \times 10^{-3} \frac{\Omega}{\mathrm{m}}$ 
$\boldsymbol{L}=\left[\begin{array}{llllll}1.927 & 1.034 & 0.993 & 0.970 & 0.861 & 0.879 \\ 1.034 & 1.927 & 0.993 & 0.861 & 0.791 & 0.810 \\ 0.993 & 0.993 & 1.929 & 0.879 & 0.810 & 0.863 \\ 0.970 & 0.861 & 0.879 & 1.927 & 1.034 & 0.993 \\ 0.861 & 0.791 & 0.810 & 1.034 & 1.927 & 0.993 \\ 0.879 & 0.810 & 0.863 & 0.993 & 0.993 & 1.929\end{array}\right] \times 10^{-6} \frac{\mathrm{H}}{\mathrm{m}}$

$\boldsymbol{G}=\left[\begin{array}{cccccc}0.200 & 0 & 0 & 0 & 0 & 0 \\ 0 & 0.200 & 0 & 0 & 0 & 0 \\ 0 & 0 & 0.200 & 0 & 0 & 0 \\ 0 & 0 & 0 & 0.200 & 0 & 0 \\ 0 & 0 & 0 & 0 & 0.200 & 0 \\ 0 & 0 & 0 & 0 & 0 & 0.200\end{array}\right] \times 10^{-12} \frac{\mathrm{S}}{\mathrm{m}}$

(6)

(7)

$\boldsymbol{C}=\left[\begin{array}{cccccc}10.272 & -2.550 & -2.000 & -1.781 & -0.881 & -0.995 \\ -2.550 & 9.702 & -2.270 & -0.881 & -0.609 & -0.677 \\ -2.000 & -2.270 & 9.627 & -0.995 & -0.677 & -1.200 \\ -1.781 & -0.881 & -0.995 & 10.272 & -2.550 & -2.000 \\ -0.881 & -0.609 & -0.677 & -2.550 & 9.702 & -2.270 \\ -0.995 & -0.677 & -1.200 & -2.000 & -2.270 & 9.627\end{array}\right]$ $\times 10^{-12} \frac{\mathrm{F}}{\mathrm{m}}$

\section{ACKNOWLEDGMENT}

The authors would like to thank the contributions of experimental participants including Yi Zhang, Jiangwu Liu of Shanxi Electric Power Company of State Grid of China, and Yanpeng Ge, Hong Zhang, Hongye Zhang of Xi'an Jiaotong University.

\section{REFERENCES}

[1] IEEE Guide for Identifying and Improving Voltage Quality in Power Systems, IEEE Standard 1250, 2011.

[2] A. Ametani, N. Nagaoka, Y. Baba, T. Ohno and K. Yamabuki, "Transients on overhead lines," in Power System Transients: Theory and Applications, 2nd ed., Boca Raton, FL, USA: CRC Press, 2016, pp. 141214.

[3] M. H. J. Bollen, E. Styvaktakis and I. Y. H. Gu, "Categorization and analysis of power system transients," IEEE Trans. Power Delivery, vol. 20, no. 3, pp. 2298-2306, Jul. 2005.

[4] IEEE Guide for Improving the Lightning Performance of Electric Power Overhead Distribution Lines, IEEE Standard 1410, 2011.

[5] C. A. Nucci and F. Rachidi, "Lightning protection of medium voltage lines," in Lightning Protection, Stevenage, UK: IET, 2009, pp. 635-680.

[6] M. M. Saha, J. Izykowski and E. Rosolowski, "Fault location-basic concept and characteristic of methods," in Fault Location on Power Networks, London, UK: Springer-Verlag London, 2010, pp. 1-27.

[7] IEEE Guide for Determining Fault Location on AC Transmission and Distribution Lines, IEEE Standard C37. 114-2014 (Revision of IEEE C37. 114-2004), 2014.

[8] W. A. Radasky, C. E. Baum and M. W. Wik, "Introduction to the special issue on high-power electromagnetics (HPEM) and intentional electromagnetic interference (IEMI)," IEEE Trans. Electromagnetic Compatibility, vol. 46, no. 3, pp. 314-321, Aug. 2004.

[9] S. Robert, "Conducted EMI issues in Smart Grids," in Conducted Electromagnetic Interference (EMI) in Smart Grids, London, UK: Springer-Verlag London, 2012, pp. 37-78.
[10] M. Paolone, C.A. Nucci, E. Petrache, and F. Rachidi, "Mitigation of lightning-induced overvoltages in medium voltage distribution lines by means of periodical grounding of shielding wires and of surge arresters: modelling and experimental validation," IEEE Trans. Power Delivery, vol. 19, no. 1, pp. 423-431, Jan. 2004.

[11] M. Omidiora and M. Lehtonen, "Mitigation of lightning flashover from tree to medium voltage aerial cable using shield wire," IEEE Trans. Power Delivery, vol. 32, no. 4, pp. 1924-1934, Aug. 2017.

[12] J. M. Gers and E. J. Holmes, Protection of Electricity Distribution Networks, 3rd ed., Stevenage, UK: IET, 2011.

[13] M. Ianoz, "Lightning and EMC," in Lightning Flash, Stevenage, UK: IET, 2003, pp. 479-502.

[14] Y. V. Parfenov, L. N. Zdoukhov, W. A. Radasky and M. Ianoz, "Conducted IEMI threats for commercial buildings," IEEE Trans. Electromagnetic Compatibility, vol. 46, no. 3, pp. 404-411, Aug. 2004.

[15] J. F. Dawson, et al., "A cost-efficient system for detecting an intentional electromagnetic interference (IEMI) attack," in Proc. International Symposium on Electromagnetic Compatibility (EMC Europe), Gothenburg, Sweden, 2014, pp. 1252-1256.

[16] F. Rachidi, M. Rubinstein, and M. Paolone, Electromagnetic Time Reversal: Application to Electromagnetic Compatibility and Power Systems, New York, NY, USA: Wiley, 2017.

[17] G. Lugrin, N. Mora Parra, F. Rachidi, M. Rubinstein and G. Diendorfer, "On the location of lightning discharges using time reversal of electromagnetic fields," IEEE Trans. Electromagnetic Compatibility, vol. 56, no. 1, pp. 149-158, Feb. 2014.

[18] R. Razzaghi, M. Scatena, M. Paolone, F. Rachidi and G. Antonini, "Locating lightning-originated flashovers in power networks using electromagnetic time reversal," presented at 2017 IPST, Seoul, Republic of Korea, 2017.

[19] R. Razzaghi, G. Lugrin, H. M. Manesh, C. Romero, M. Paolone, and F. Rachidi, "An efficient method based on the electromagnetic time reversal to locate faults in power networks," IEEE Trans. Power Delivery, vol. 28, no. 3, pp. 1663-1673, Jul. 2013.

[20] R. Razzaghi, M. Paolone, F. Rachidi, J. Descloux, B. Raison, and N. Retiere, "Fault location in multi-terminal HVDC networks based on electromagnetic time reversal with limited time reversal window," presented at 2014 PSCC, Wroclaw, Poland, 2014.

[21] R. Razzaghi, F. Rachidi and M. Paolone, "Single-End FPGA-based fault location system for radial/meshed AC/DC networks based on the electromagnetic time reversal theory," presented at 2017 IEEE PowerTech, Manchester, UK, 2017.

[22] A. Codino, Z. Wang, R. Razzaghi, M. Paolone, and F. Rachidi, "An alternative method for locating faults in transmission line networks based on time reversal," IEEE Trans. Electromagnetic Compatibility, vol. 59, no. 5, pp. 1601-1612, Oct. 2017.

[23] Z. Wang, A. Codino, R. Razzaghi, M. Paolone, and F. Rachidi, "Using electromagnetic time reversal to locate faults in transmission lines: definition and application of the "mirrored minimum energy" property," presented at 2017 International Symposium on Electromagnetic Compatibility (EMC Europe), Angers, France, 2017.

[24] M. Kafal, A. Cozza and L. Pichon, "Locating multiple soft faults in wire networks using an alternative DORT implementation," IEEE Trans. Instrumentation and Measurement, vol. 65, no. 2, pp. 399-406, Feb. 2016.

[25] M. Kafal, A. Cozza and L. Pichon, "Locating faults with high resolution using single-frequency TR-MUSIC processing," IEEE Trans. Instrumentation and Measurement, vol. 65, no. 10, pp. 2342-2348, Oct. 2016.

[26] M. Rubinstein, F. Rachidi and M. Paolone, "Time reversal: a different perspective," in Electromagnetic Time Reversal: Application to Electromagnetic Compatibility and Power Systems, New York, NY, USA: Wiley, 2017, pp. 1-28.

[27] M. Fink, "Time reversal of ultrasonic fields - I: basic principles," IEEE Trans. Ultrasonics, Ferroelectrics and Frequency Control, vol. 39, no. 5, pp. 555-566, Sept. 1992.

[28] F. Wu, J. L. Thomas, and M. Fink, "Time reversal of ultrasonic fields II: experimental results," IEEE Trans. Ultrasonics, Ferroelectrics and Frequency Control, vol. 39, no. 5, pp. 567-578, Sept. 1992.

[29] D. Cassereau and M. Fink, "Time reversal of ultrasonic fields - III: theory of the closed time reversal cavity," IEEE Trans. Ultrasonics, Ferroelectrics and Frequency Control, vol. 39, no. 5, pp. 579-592, Sept. 1992.

[30] R. Razzaghi, G. Lugrin, F. Rachidi and M. Paolone, "Assessment of the influence of losses on the performance of the electromagnetic time 
reversal fault location method," IEEE Trans. Power Delivery, vol. 32, no. 5, pp. 2303-2312, Oct. 2017.

[31] V. Cooray and M. Fernando, "Lightning parameters of engineering interest," in Lightning Protection, Stevenage, UK: IET, 2003, pp. 15-96.

[32] IEEE Guide for the Application of Transient Recovery Voltage for AC High-Voltage Circuit Breakers, IEEE Standard C37. 011-2011 (Revision of IEEE C37. 011-2005), 2011.

[33] A. Greenwood, Electrical Transients in Power Systems, 2nd ed., New York, NY, USA: Wiley, 1991.

[34] H. W. Dommel, "Digital computer solution of electromagnetic transients in single- and multiphase networks," IEEE Trans. Power Apparatus and Systems, vol. PAS-88, pp. 388-399, Apr. 1969.

[35] J. Mahseredjian, S. Dennetière, L. Dubé, B. Khodabakhchian and L. Gérin-Lajoie, "On a new approach for the simulation of transients in power systems," Electric Power Systems Research, vol. 77, no.11, pp. 1514-1520, Sept. 2007.

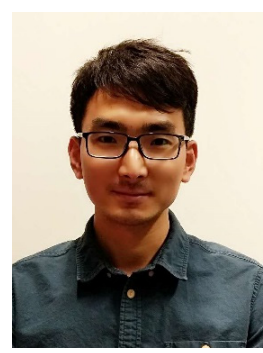

Zhaoyang Wang (MS'17) received the M.Sc. degree in electrical engineering from Xi'an Jiaotong University, Xi'an, China, in 2015, and is currently pursuing the $\mathrm{Ph} . \mathrm{D}$. degree in electrical engineering at the Electromagnetic Compatibility Laboratory of the Swiss Federal Institute of Technology of Lausanne (EPFL), Lausanne, Switzerland.

His research interests include electromagnetic transients and power system protection.

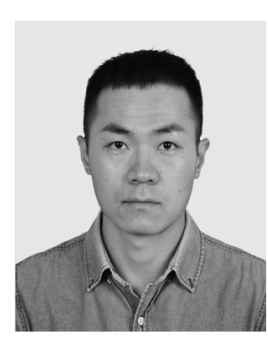

Shaoyin He received the M. Sc. degree in electronic engineering from Xidian University, Shaanxi, China, in 2011. He is currently working towards the Ph.D. degree in electrical engineering at Xi'an Jiaotong University, Shaanxi, China.

His research interests include electromagnetic transients and fault location techniques.

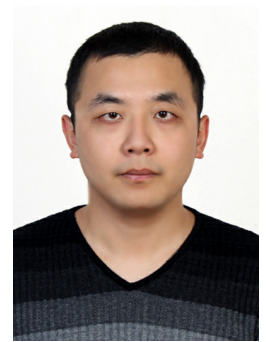

Qi Li was born in Shannxi, China, in 1993. He received the B.Sc. degree in measurement techniques and instrumentation from Xi'an Jiaotong University, Xi'an, China, in 2015 and is currently pursuing the M. Sc. degree in electrical engineering.

His research interests include the transient electromagnetic measurement.

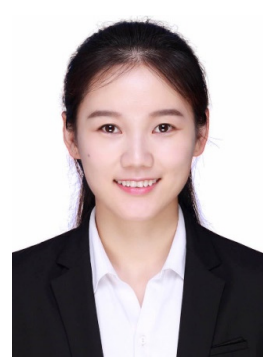

Buying Liu is currently working towards the M.Sc. degree in software engineering at Xi'an Jiaotong University, Shaanxi, China.

Her research interests include electromagnetic transients in power system and software modeling.

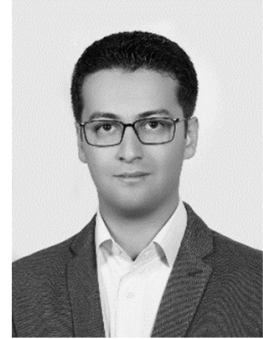

Reza Razzaghi (S'10-M'17) received the B.Sc. and M.Sc. degrees from the University of Tehran, Tehran, Iran, in 2009 and 2011, respectively, and the Ph.D. degree from the Swiss Federal Institute of Technology of Lausanne (EPFL), Lausanne, Switzerland, in 2016, all in electrical engineering. He is currently a Lecturer (Assistant Professor) with the Department of Electrical and Computer Systems Engineering, Monash University, Melbourne, Australia.

His research interests include power system protection and control, stability analysis, phasor measurement units, and power system transients. Dr Razzaghi has been the recipient of numerous awards including the Distinguished Doctorate Award of EPFL and Basil Papadias Best Paper Award at IEEE PowerTech conference in 2013.

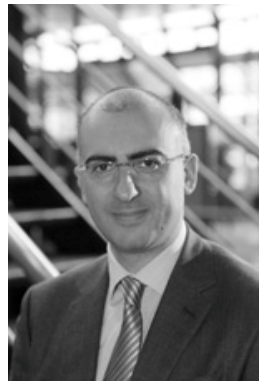

Mario Paolone (M'07-SM'10) received the M.Sc. (with Hons.) and Ph.D. degrees in electrical engineering from the University of Bologna, Bologna, Italy, in 1998 and 2002, respectively. In 2005, he was appointed as an Assistant Professor in power systems with the University of Bologna, where he was with the Power Systems Laboratory until 2011. In 2010, he received the Associate Professor eligibility from the Polytechnic of Milan, Italy. Since 2011, he joined the Swiss Federal Institute of Technology, Lausanne, Switzerland, where he is currently Full Professor, Chair of the Distributed Electrical Systems Laboratory, and Head of the Swiss Competence Center for Energy Research Future Swiss Electrical infrastructure.

$\mathrm{He}$ has authored or co-authored over 230 scientific papers published in reviewed journals and international conferences. His current research interests include power systems with particular reference to real-time monitoring and operation, power system protections, power systems dynamics, and power system transients.

Dr. Paolone was the Co-Chairperson of the Technical Program Committees of the 9th edition of the International Conference of Power Systems Transients (2009) and the 2016 Power Systems Computation Conference. He is the Chair of the Technical Program Committee of the 2018 Power Systems Computation Conference. In 2013, he was a recipient of the IEEE EMC Society Technical Achievement Award. He has co-authored several papers that received the following awards: the Best IEEE Trans. on Electromagnetic Compatibility Paper Award in 2017, the Best Paper Award at the 13th International Conference on Probabilistic Methods Applied to Power Systems, Durham, U.K., in 2014, the Basil Papadias Best Paper Award at the 2013 IEEE PowerTech, Grenoble, France, and the Best Paper Award at the International Universities Power Engineering Conference in 2008

$\mathrm{He}$ is the Editor-in-Chief of the journal Sustainable Energy, Grids and Networks (Elsevier) and an Associate Editor of the IEEE Trans. on Industrial Informatics.

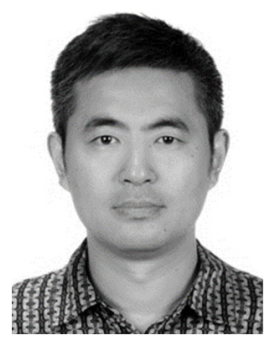

Yanzhao Xie (M'12) was born in 1973. He received the Ph.D. degree in electrical engineering from Tsinghua University, Beijing, China, in December, 2005

He is currently a Professor of School of Electrical Engineering, Xi'an Jiaotong University, China. His research interests include electromagnetic compatibility, electromagnetic transients in power system and high-power electromagnetics, etc.

$\mathrm{He}$ has been the director of national center for international research on transient electromagnetics and applications (TEA) since 2016

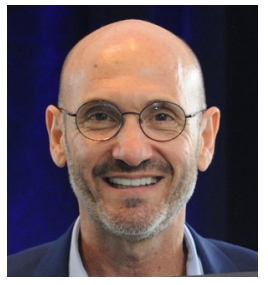

Marcos Rubinstein (M'84-SM'11-F'14) received the Master's and Ph.D. degrees in electrical engineering from the University of Florida, Gainesville, FL, USA, in 1986 and 1991, respectively. In 1992, he joined the Swiss Federal Institute of Technology, Lausanne, Switzerland, where he was involved in the fields of electromagnetic compatibility and lightning. In 1995, he was with Swisscom, where he worked in numerical electromagnetics and EMC. In 2001, he moved to the University of Applied Sciences of Western Switzerland HES-SO, Yverdon-les-Bains, where he is currently a full Professor, head of the advanced Communication Technologies Group and a member of the IICT Institute Team.

$\mathrm{He}$ is the author or coauthor of more than 200 scientific publications in reviewed journals and international conferences. He is also the coauthor of seven book chapters. He is the Chairman of the International Project on Electromagnetic Radiation from Lightning to Tall structures, served as the Editor-in-Chief of the Open Atmospheric Science Journal, and currently serves as an Associate Editor of the IEEE Transactions on Electromagnetic Compatibility.

Prof. Rubinstein received the best Master's Thesis award from the University of Florida. He received the IEEE achievement award, and he is a corecipient of the NASA's Recognition for Innovative Technological Work award. 
He is a Fellow of the IEEE and of the SUMMA Foundation, a member of the Swiss Academy of Sciences and of the International Union of Radio Science.

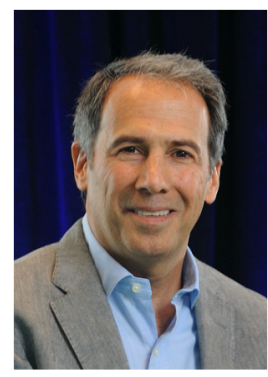

Farhad Rachidi (M'93-SM'02-F'10) received the M.S. degree in electrical engineering and the Ph.D. degree from the Swiss Federal Institute of Technology, Lausanne, Switzerland, in 1986 and 1991, respectively. He was with the Power Systems Laboratory, Swiss Federal Institute of Technology, until 1996. In 1997, he joined the Lightning Research Laboratory, University of Toronto, Toronto, ON, Canada. From 1998 to 1999 , he was with Montena EMC, Rossens, Switzerland. He is currently a Titular Professor and the Head of the EMC Laboratory with the Swiss Federal Institute of Technology, Lausanne, Switzerland. He has authored or co-authored over 170 scientific papers published in peer-reviewed journals and over 350 papers presented at international conferences.

Dr. Rachidi is currently a member of the Advisory Board of the IEEE Transactions on Electromagnetic Compatibility and the President of the Swiss National Committee of the International Union of Radio Science. He has received numerous awards including the 2005 IEEE EMC Technical Achievement Award, the 2005 CIGRE Technical Committee Award, the 2006 Blondel Medal from the French Association of Electrical Engineering, Electronics, Information Technology and Communication (SEE), the 2016 Berger Award from the International Conference on Lightning Protection, the 2016 Best Paper Award of the IEEE Transactions on EMC, and the 2017 Motohisa Kanda Award for the most cited paper of the IEEE Transactions on EMC. In 2014, he was conferred the title of Honorary Professor of the Xi'an Jiaotong University in China. He served as the Vice-Chair of the European COST Action on the Physics of Lightning Flash and its Effects from 2005 to 2009, the Chairman of the 2008 European Electromagnetics International Symposium, the President of the International Conference on Lightning Protection from 2008 to 2014, the Editor-in-Chief of the Open Atmospheric Science Journal (2010-2012) and the Editor-in-Chief of the IEEE Transactions on Electromagnetic Compatibility from 2013 to 2015. 